\title{
BMJ Open PRactice of VENTilation in Middle- Income Countries (PRoVENT-iMIC): rationale and protocol for a prospective international multicentre observational study in intensive care units in Asia
}

Luigi Pisani, ${ }^{1,2}$ Anna Geke Algera, ${ }^{1}$ Ary Serpa Neto, ${ }^{1,3}$ Areef Ahsan, ${ }^{4}$ Abigail Beane, ${ }^{2}$ Kaweesak Chittawatanarat, ${ }^{5}$ Abul Faiz, ${ }^{2,6}$ Rashan Haniffa, ${ }^{2}$ Reza Hashemian, ${ }^{7}$ Madiha Hashmi, ${ }^{8}$ Hisham Ahmed Imad, ${ }^{9}$ Kanishka Indraratna, ${ }^{10}$ Shivakumar Iyer, ${ }^{11}$ Gyan Kayastha, ${ }^{12}$ Bhuvana Krishna, ${ }^{13}$ Hassan Moosa, ${ }^{14}$ Behzad Nadjm, ${ }^{15}$ Rajyabardhan Pattnaik, ${ }^{16}$ Sriram Sampath, ${ }^{13}$ Louise Thwaites, ${ }^{17} \mathrm{Ni}$ Ni Tun, ${ }^{18}$ Nor'azim Mohd Yunos, ${ }^{19}$ Salvatore Grasso, ${ }^{20}$ Frederique Paulus, ${ }^{1}$ Marcelo Gama de Abreu, ${ }^{21}$ Paolo Pelosi, ${ }^{22}$ Arjen M Dondorp, ${ }^{1,2}$ Marcus J Schultz, ${ }^{1,2,23}$ for the PRoVENT-iMIC investigators, MORU and the PROVE network

To cite: Pisani L, Algera AG, Serpa Neto $A$, et al. PRactice of VENTilation in Middle-Income Countries (PRoVENT-iMIC): rationale and protocol for a prospective internationa multicentre observational study in intensive care units in Asia. BMJ Open 2018:8:e020841. doi:10.1136/ bmjopen-2017-020841

- Prepublication history for this paper is available online. To view these files please visit the journal online (http://dx.doi org/10.1136/bmjopen-2017020841).

Received 27 November 2017 Revised 19 March 2018 Accepted 20 March 2018
Check for updates

For numbered affiliations see end of article.

Correspondence to

Dr Luigi Pisani;

luigipisani@gmail.com

\section{ABSTRACT}

Introduction Current evidence on epidemiology and outcomes of invasively mechanically ventilated intensive care unit (ICU) patients is predominantly gathered in resource-rich settings. Patient casemix and patterns of critical illnesses, and probably also ventilation practices are likely to be different in resourcelimited settings. We aim to investigate the epidemiological characteristics, ventilation practices and clinical outcomes of patients receiving mechanical ventilation in ICUs in Asia. Methods and analysis PRoVENT-iMIC (study of PRactice of VENTilation in Middle-Income Countries) is an international multicentre observational study to be undertaken in approximately 60 ICUs in 11 Asian countries. Consecutive patients aged 18 years or older who are receiving invasive ventilation in participating ICUs during a predefined 28-day period are to be enrolled, with a daily follow-up of 7 days. The primary outcome is ventilatory management (including tidal volume expressed as $\mathrm{mL} / \mathrm{kg}$ predicted body weight and positive end-expiratory pressure expressed as $\mathrm{cm} \mathrm{H}_{2} \mathrm{O}$ ) during the first 3 days of mechanical ventilation-compared between patients at no risk for acute respiratory distress syndrome (ARDS), patients at risk for ARDS and in patients with ARDS (in case the diagnosis of ARDS can be made on admission). Secondary outcomes include occurrence of pulmonary complications and allcause ICU mortality.

Ethics and dissemination PRoVENT-iMIC will be the first international study that prospectively assesses ventilation practices, outcomes and epidemiology of invasively ventilated patients in ICUs in Asia. The results of this large study, to be disseminated through conference presentations and publications in international peer-reviewed journals, are of ultimate importance when designing trials of invasive ventilation in resource-limited ICUs. Access to source data will be made available through national or international

\section{Strengths and limitations of this study}

- PRactice of VENTilation in Middle-Income Countries is an international multicentre observational study with a wide representation of Asian countries, allowing inferences on epidemiology, management and outcomes of mechanical ventilation across the entire subcontinent.

- The attention on ventilation practice will provide robust data on this specific domain while the 7 days follow-up will allow precise recording of pulmonary complications at their origin.

- The study will have a sample size large enough to obtain precise estimates of pulmonary complications and intensive care unit mortality and to examine potential associations between ventilation practice and these outcomes.

- One limitation is the potential constraint of laboratory data, generating a limited dataset not comprising daily severity scores useful for statistical controlling purposes.

- The conceivable limitation in blood gas analysis and imaging examinations may limit the documentation of insurgence or worsening of acute respiratory distress syndrome and other pulmonary complications.

anonymised datasets on request and after agreement of the PRoVENT-iMIC steering committee.

Trial registration number NCT03188770; Pre-results.

\section{INTRODUCTION}

Invasive mechanical ventilation is a frequently applied intervention in patients in intensive 
care units (ICUs) and a mandatory strategy in patients under general anaesthesia for surgery. There is increased understanding how invasive ventilation can harm the lungs, in ICU patients with the acute respiratory distress syndrome (ARDS), ${ }^{1}$ as well as in ICU patients with less injured or uninjured lungs, and in surgery patients who usually have healthy lungs. ${ }^{2} \mathrm{~A}$ central cause is that invasive ventilation with positive pressure may overdistend one lung area while failing to recruit another, compromising gas exchange but also, and more importantly, increasing or inducing pulmonary injury. There is convincing evidence that this harm can be partly prevented by adjusting volume and pressure settings on the ventilator. Indeed, use of low tidal volumes $\left(\mathrm{V}_{\mathrm{T}}\right),{ }^{3-5}$ to prevent overdistension, and sufficient positive end-expiratory pressure (PEEP), ${ }^{356}$ to prevent alveolar collapse or atelectrauma, have both been found to improve outcomes of various types of patients, and their use is increasingly recommended ${ }^{7-9}$ Furthermore, the driving pressure seems to be another key variable in the development of injury caused by mechanical ventilation, as a large individual patient data meta-analysis showed a clear and consistent association between driving pressure and mortality. ${ }^{10}$

Practice of invasive ventilation has evolved over time, with a more extensive use of ventilator settings that are proven to prevent against so-called ventilator-induced lung injury. The recent LUNG SAFE ('Large observational study to UNderstand the Global impact of Severe Acute respiratory Failure') showed that by now up to two in every three patients with ARDS receive so-called lung-protective ventilation. ${ }^{11}$ Results of PRoVENT ('PRactice of VENTilation in critically ill patients without ARDS at onset of ventilation study') are in line with those from LUNG SAFE, showing that one in every two ICU patients without ARDS receive ventilation with lung-protective settings. ${ }^{12}$ Results of LAS VEGAS ('Local ASsessment of VEntilatory Management During General Anaesthesia for Surgery study') even suggests increased use of lung-protective ventilation in the operating rooms. ${ }^{13}$ It should be noticed, though, that LUNG SAFE, PRoVENT and LAS VEGAS were mainly performed in high-income countries, and exclusively recruiting patients in resource-rich centres, which limits the generalisability of their results to lower-income countries and resource-limited settings. Historical descriptions of cohorts of invasively ventilated patients in resource-poor settings have been published, but these were all small in size, and while suggesting the existence of ventilator-related deaths they largely failed to report key ventilator parameters. ${ }^{14-16}$ Continued use of high $\mathrm{V}_{\mathrm{T}}$ has been reported in a recent Brazilian study, ${ }^{17}$ while a study from India suggests a change towards the use of lower $\mathrm{V}_{\mathrm{T}}{ }^{18}$

There are several reasons to consider important differences with regard to practice of ventilation between resource-rich and resource-limited settings. The disparity in resources may limit the availability as well as the safety of certain ventilator settings. ${ }^{19}$ Awareness of the impact of invasive ventilation on lung tissue, and the benefit of using lung-protective ventilation settings could be severely limited. ${ }^{20} \mathrm{~V}_{\mathrm{T}}$ and PEEP may be poorly titrated due to insufficient staffing, and due to the absence of arterial blood gas monitoring, pulse oximetry or capnography. ${ }^{21}$ Other reasons not to implement use of low $\mathrm{V}_{\mathrm{T}}$ and sufficient levels of PEEP include alleged side effects associated with their use, like the need for higher respiratory rates, increased sedation requirements and even the promotion of patient-ventilator asynchrony. As invasive ventilation with higher PEEP may cause haemodynamic instability, limited access to fluids and vasoactive drugs may hamper its use. Finally, as resource-poor ICUs are usually situated in tropical countries their casemix and indications for invasive ventilation are strikingly different. ${ }^{18}$

To gain a better insight into the ventilation practice, outcomes and epidemiological characteristics of ICU patients receiving invasive ventilation in resource-limited settings, we plan to perform the PRoVENT-iMIC ('Practice of VENTilation in Middle-Income Countries study'), a prospective observational cohort study in ICUs in Asia. We also aim to describe the association between certain ventilator settings and patient-centred outcomes. We hypothesise that practice of ventilation is highly variable, in particular with respect to $\mathrm{V}_{\mathrm{T}}$ and PEEP settings. This understanding is fundamental to planning any intervention study in these countries in the future.

\section{METHODS AND ANALYSIS \\ Design and setting}

PRoVENT-iMIC is an international multicentre observational study in consecutive ICU patients receiving invasive mechanical ventilation during a 28-day period, expected to run in approximately 60 centres in the following Asian countries: Thailand, Vietnam, Myanmar, Pakistan, Nepal, Bangladesh, Malaysia, Sri Lanka, Maldives, Iran and India. These countries belong to the low-income or middle-income economies, as classified by the World Bank. ${ }^{22}$ PRoVENT-iMIC is conducted in accordance with the Declaration of Helsinki and is registered at www.clinicaltrials.gov (trial identification number NCT 03188770). Figure 1 shows the study flow chart.

\section{Study population}

Consecutive patients intubated for ventilation during a predefined period of 28 days are enrolled. Inclusion is not restricted to patients who are intubated in the ICU, as also patients who started invasive ventilation in the emergency room, normal ward, community or operating room directly preceding the present ICU admission are eligible for participation, without any minimum or maximum hours of ventilation needed for inclusion. The exclusion criteria include age $<18$ years, use of non-invasive ventilation not followed by invasive ventilation, patients whose invasive mechanical ventilation started before the 28-day period of inclusion, and patients transferred from another hospital under invasive ventilation. 
All adult ICU-patients in need of MV in a 28 day period

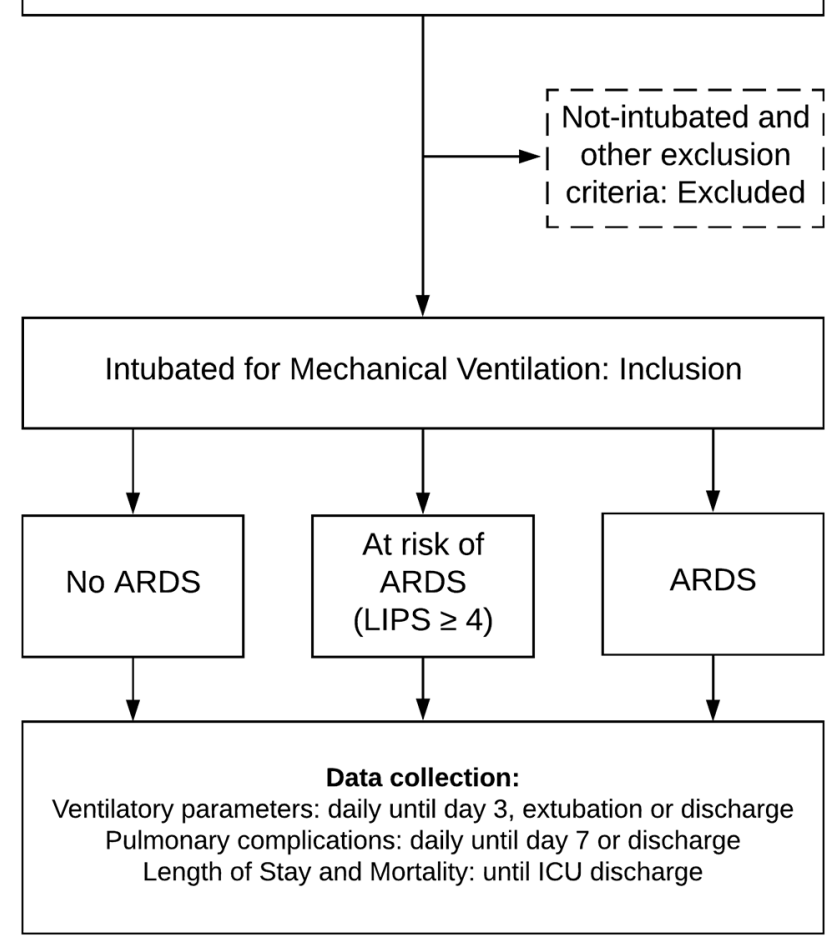

Figure 1 Flow chart of inclusion of PRoVENT-iMIC. ARDS, acute respiratory distress syndrome; ICU, intensive care unit; LIPS, Lung Injury Prediction Score; MV, mechanical ventilation; PRoVENT-iMIC, PRactice of VENTilation in Middle-Income Countries.

Patients will be stratified in three groups for comparison of the primary and secondary endpoints: patients without ARDS, patients without but at risk for development of ARDS, according to the Lung Injury Prediction Score (LIPS, table 1) ${ }^{23}$ and patients with ARDS, according to the Berlin definition. ${ }^{24}$ Patients with ARDS will also be stratified according to severity of ARDS, based on the oxygenation (mild, moderate and severe ARDS categories).

\section{Study conduct}

Local investigators will screen all patients who start invasive ventilation in one of the participating ICUs during a predefined period of 28 days, lasting from 08:00 hours on the Monday of the first week to 07:59 hours on the Monday 4 weeks later. The exact starting date will be flexible for participating centres and shall be determined by the national study coordinator. Data collection has started in November 2017 in some sites; all sites are expected to initiate the service evaluation within 1 year after the overall start.

\section{Data to be collected}

Baseline and demographic variables will be collected on the day of admission, including gender, age, actual or estimated weight and height, smoking status, comorbidities including chronic obstructive pulmonary disease,

\begin{tabular}{|c|c|c|c|}
\hline $\begin{array}{l}\text { Predisposing } \\
\text { conditions }\end{array}$ & Score & Risk modifiers & Score \\
\hline Shock & 2 & Alcohol abuse & 1 \\
\hline Aspiration & 2 & $\mathrm{BMI}>30 \mathrm{~kg} / \mathrm{m}^{2}$ & 1 \\
\hline Sepsis & 1 & Hypoalbuminaemia & 1 \\
\hline Pneumonia & 1.5 & Chemotherapy & 1 \\
\hline High-risk surgery & & $\mathrm{FiO}_{2}>0.35(>4 \mathrm{~L} / \mathrm{min})$ & 2 \\
\hline Orthopaedic spine & 1 & $\mathrm{RR}>30 \mathrm{bpm}$ & 1.5 \\
\hline Acute abdomen & 2 & $\mathrm{SpO}_{2}<95 \%$ & 1 \\
\hline Cardiac & 2.5 & Acidosis $(\mathrm{PH}<7.35)$ & 1.5 \\
\hline Aortic vascular & 3.5 & Diabetes mellitus* & -1 \\
\hline $\begin{array}{l}\text { Emergency } \\
\text { surgery }\end{array}$ & 1.5 & & \\
\hline \multicolumn{4}{|l|}{ High-risk trauma } \\
\hline $\begin{array}{l}\text { Traumatic brain } \\
\text { injury }\end{array}$ & 2 & & \\
\hline Smoke inhalation & 2 & & \\
\hline Near drowning & 2 & & \\
\hline Lung contusion & 1.5 & & \\
\hline Multiple fractures & 1.5 & & \\
\hline
\end{tabular}

${ }^{*}$ To consider only in patients with sepsis.

$\mathrm{BMI}$, body mass index; $\mathrm{FiO}_{2}$, inspired oxygen fraction; RR, respiratory rate; $\mathrm{SpO}_{2}$, pulse oximetry oxygen saturation.

active cancer, heart failure, diabetes mellitus, chronic kidney failure, liver cirrhosis and arterial hypertension, the presence of ARDS according to the Berlin definition, the LIPS, reason for ICU admission. On the day of start of invasive ventilation, we will document the reason for starting mechanical ventilation and whether the patient received non-invasive ventilation before intubation.

Every day, until day 3 from admission in the ICU, until ICU discharge or death, whichever comes first, the ventilation status and ventilation characteristics will be collected, including ventilation mode, $\mathrm{V}_{\mathrm{T}}$ size, respiratory rate (set and measured), peak and plateau pressure, PEEP, inspired oxygen fraction, peripheral oxygen saturation, blood gas analysis data when available (arterial oxygen tension, arterial carbon dioxide tension, arterial bicarbonate, arterial $\mathrm{pH}$ ), end-tidal $\mathrm{CO}_{2}$, when available and haemodynamic parameters like heart rate and systolic blood pressure.

Every day, until day 7, ICU discharge or death, whichever comes first, the occurrence of pulmonary complications will be scored, including new requirement of invasive ventilation after initial extubation, pulmonary infections, atelectasis, pneumothorax, pleural effusions, new pulmonary infiltrates and development or worsening of ARDS.

On the day of ICU discharge (maximum 60 days after recruitment), outcome will be recorded as follows: death, discharge to ward, to medium care or high dependency 


\begin{tabular}{|c|c|c|c|}
\hline Criteria & \multicolumn{3}{|l|}{ Definition } \\
\hline Time & \multicolumn{3}{|c|}{ Within 1 week of a known clinical insult or new/worsening respiratory symptoms. } \\
\hline Chest imaging* & \multicolumn{3}{|c|}{ Bilateral opacities not fully explained by effusions, lobar/lung collapse or nodules. } \\
\hline Origin of oedema & \multicolumn{3}{|c|}{$\begin{array}{l}\text { Respiratory failure not fully explained by cardiac failure or fluid overload; need objective assessmen } \\
\text { to exclude hydrostatic oedema if no risk factors present (eg, echocardiography). }\end{array}$} \\
\hline Oxygenationt & 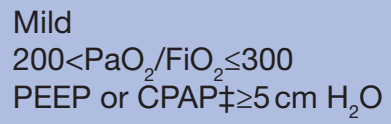 & $\begin{array}{l}\text { Moderate } \\
100<\mathrm{PaO}_{2} / \mathrm{FiO}_{2} \leq 200 \\
\text { PEEP } \geq 5 \mathrm{~cm} \mathrm{H}_{2} \mathrm{O}\end{array}$ & $\begin{array}{l}\text { Severe } \\
\mathrm{PaO}_{2} / \mathrm{FiO}_{2} \leq 100 \\
\mathrm{PEEP} \geq 5 \mathrm{~cm} \mathrm{H}_{2} \mathrm{O}\end{array}$ \\
\hline
\end{tabular}

${ }^{*}$ Chest X-ray or CT scan.

†lf altitude higher than $1000 \mathrm{~m}$ correction factor should be made as follows: $\mathrm{PaO}_{2} / \mathrm{FiO}_{2} \mathrm{x}($ barometric pressure/760).

$\ddagger$ This may be delivered non-invasively in the mild ARDS.

ARDS, acute respiratory distress syndrome; CPAP, continuous positive airway pressure; $\mathrm{FiO}_{2}$, fractional inspired oxygen; $\mathrm{PaO}{ }_{2}$, arterial oxygen tension; PEEP, positive end-expiratory pressure.

unit, discharge to home for palliative care, or transfer to another ICU. The date of extubation, reintubation and tracheostomy (if performed) will also be recorded in this moment.

\section{Study endpoints}

The primary endpoint is $\mathrm{V}_{\mathrm{T}}$ size in millilitres per kilogram of predicted body weight $(\mathrm{mL} / \mathrm{kg}$ PBW) and PEEP in centimetres of water $\left(\mathrm{cm} \mathrm{H}_{2} \mathrm{O}\right)$ used among diverse ICU patient categories during the first 3 days of mechanical ventilation. Secondary clinical endpoints include other ventilation parameters (including respiratory system driving pressure, the proportion of patients at risk of ARDS as stratified by the LIPS or ARDS defined by the Berlin definition, the occurrence of pulmonary complications, length of stay in ICU, duration of invasive ventilation and all-cause ICU mortality.

\section{Definitions}

All-cause ICU mortality is defined as any death in the ICU. ICU length of stay is defined as the time between ICU admission and ICU discharge or death in ICU. The number of days of ventilation is defined as time between endotracheal intubation and successful extubation (in case of intermittent mechanical ventilation via a tracheostomy, every day a patient needs ventilation counts as one extra day, irrespective of the duration of ventilation on that specific day). In case of non-invasive ventilation, the duration will be assessed separated from the assessment of invasive ventilation. The presence of spontaneous activity will be identified by any recorded difference between the set and measured respiratory rate.

Driving pressure will be calculated by subtracting the level of PEEP from the plateau pressure (Pplat in volume-control ventilation) or maximal airway pressure (Pmax in pressure-control ventilation). Pplat and Pmax are considered reliable for this calculation if the patient is receiving complete ventilatory assistance without evidence of spontaneous activity, that is, only when the set respiratory rate equals the measured respiratory rate. Peak airways pressures will not be used to compute driving pressure as these represent a poor surrogate of the plateau pressure. Only pulmonary complications that occur after the first 24 hours of invasive ventilation will be considered in analysis, as events preceding this time point may very well be considered the potential reason for intubation. A pulmonary infection requires the presence of new or changed lung opacities on chest radiography and/or new or changed sputum plus at least a temperature $>38.3^{\circ} \mathrm{C}$ or a white cell count $>12 \times 10^{\wedge} 9 / \mathrm{L}$ of blood. Atelectasis require the presence of increased density (lung opacity) on one or more chest radiographs with displacement of the fissures towards the area of atelectasis, crowding of pulmonary vessels and bronchi in the atelectatic region, upward displacement of hemidiaphragm ipsilateral to the side of atelectasis, that may be accompanied by shift of the mediastinum or hilum towards the affected area and compensatory overinflation in the unaffected lung. ${ }^{25}$ Pleural effusion is suggested by lung opacification with shift of the mediastinum, hilum or hemidiaphragm towards the non-affected area. Pneumothorax requires the presence of air in the pleural space with no vascular bed surrounding the visceral pleura. ARDS is defined according to the Berlin definition ${ }^{24}$ with alternative oxygenation criteria based on pulse oximetry oxygen saturation/fractional inspired oxygen

Table 2B Alternative oxygenation criteria (if $\mathrm{PaO}_{2}$ data unavailable)

$\begin{array}{llll}\text { Criteria } & \text { Mild ARDS } & \text { Moderate ARDS } & \text { Severe ARDS } \\ \text { Oxygenation } & 235<\mathrm{SpO}_{2} / \mathrm{FiO}_{2} \leq 315 & 150<\mathrm{SpO}_{2} / \mathrm{FiO}_{2} \leq 235 & \mathrm{SpO}_{2} / \mathrm{FiO}_{2} \leq 150 \\ & \text { PEEP or CPAP } \geq 5 \mathrm{~cm} \mathrm{H}_{2} \mathrm{O} & \mathrm{PEEP} \geq 5 \mathrm{~cm} \mathrm{H}_{2} \mathrm{O} & \mathrm{PEEP} \geq 5 \mathrm{~cm} \mathrm{H} \mathrm{H}_{2} \mathrm{O}\end{array}$

ARDS, acute respiratory distress syndrome; $\mathrm{CPAP}$, continuous positive airway pressure; $\mathrm{FiO}_{2}$, fractional inspired oxygen; $\mathrm{PaO}{ }_{2}$, arterial oxygen tension; $\mathrm{SpO}_{2}$, pulse oximetry oxygen saturation; PEEP, positive end-expiratory pressure. 


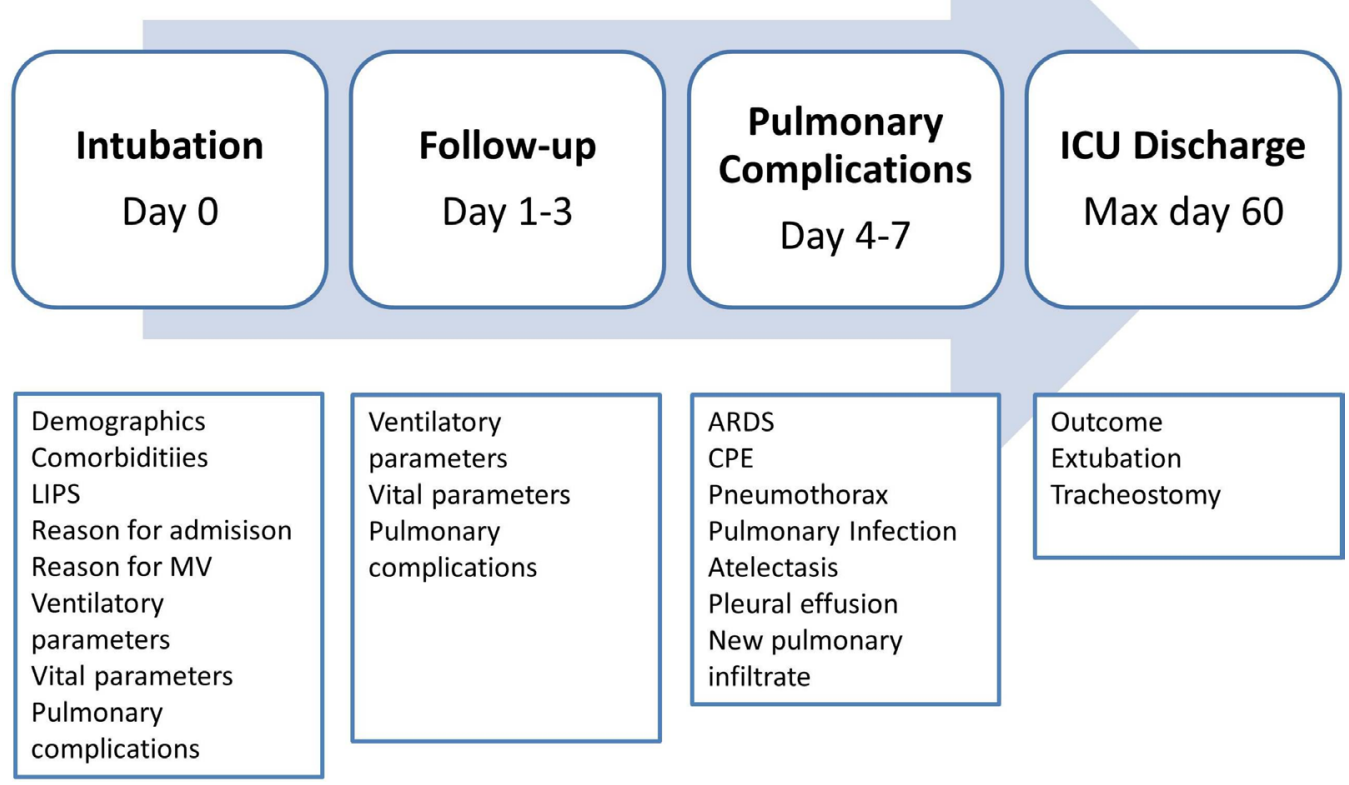

Figure 2 Sequence of data submission in the electronic case report form. ARDS, acute respiratory distress syndrome; CPE, cardiogenic pulmonary oedema; ICU, intensive care unit; LIPS, Lung Injury Prediction Score; MV, mechanical ventilation.

applicable only when blood gas analysis data is unavailable (table 2A,B). ${ }^{26}{ }^{27}$ Worsening of ARDS is defined as any change in the prior classification (ie, from mild to moderate or severe ARDS, or from moderate to severe ARDS).

\section{Data management}

Data will be collected from a paper medical chart, or an electronic patient data management system if available. Local investigators transcribe the collected data directly onto an anonymised internet-based electronic case report form (CRF) (Research Electronic Data Capture, ${ }^{28}$ www.projectredcap.org). In some centres, data may be recorded on paper CRF and successively transcribed on the electronic CRF at a later time point. Access to the data-entry system is protected by a personalised username and password. The data will be kept on a central secured server located at the Hospital Israelita Albert Einstein, Sao Paulo, Brazil. The structure of the electronic CRF is detailed in figure 2. A screening log with limited patient data will be completed with all the included and excluded patients during the enrolment window. Participating centres are instructed to enter data for the daily follow-up using values obtained as close as possible to 08:00 hours, but only when the patient is stable at that time point. The study day for the recording of pulmonary complications will be defined as the natural 24 hours period from 00:00 to 23:59 hours, to ensure that data are captured only once. Data for ICU discharge will be collected until a maximum of 60 days after ICU admission, after which the CRF for that patient will be closed.

\section{Study sites}

PRoVENT-iMIC will be conducted in 11 Asian countries, with a varying number of ICUs per country. Participating ICUs are selected on the basis of willingness to participate. There are no a priori established requirements for participation, and private as well as public centres are eligible to represent real-life practices. A one-time web-based prestudy survey on structure, organisational aspects and delivery of care in the participating centres will be performed. Each participating centre is surveyed once regarding the following information: hospital characteristics (private vs public), ICU characteristics (medical vs surgical vs mixed and open vs closed, number of ICU beds, annual number of patient admitted, number of ventilators available, and other organ support measures) and staffing (nurse-to-patient ratio, physician-to-patient ratio, presence of specialised medical staff and overnight coverage).

\section{Statistical analysis plan}

No formal sample size calculation was performed, but we expect each centre to enrol 20-40 patients in the allocated time period, yielding a total of 1200-2400 patients. We consider this figure sufficient to analyse the study endpoints.

Normally distributed variables will be expressed by their mean and SD; not normally distributed variables will be expressed by their medians and IQR; categorical variables will be expressed as $\mathrm{n}(\%)$. In test groups of continuous normally distributed variables, Student's t-test will be used. Likewise if continuous data are not normally 
distributed, the Mann-Whitney U test will be used. Categorical variables will be compared with the $\chi^{2}$ test or Fisher's exact test or when appropriate as relative risks. Statistical uncertainty will be expressed by $95 \%$ CIs.

The primary outcome $\left(\mathrm{V}_{\mathrm{T}}\right.$ size $(\mathrm{mL} / \mathrm{kg} \mathrm{PBW})$ and PEEP $\left(\mathrm{cm} \mathrm{H}_{2} \mathrm{O}\right)$ levels during the first 3 days of mechanical ventilation) will be analysed and compared between patients at no risk for ARDS, patients at risk for ARDS and in patients with ARDS (in case the diagnosis of ARDS could be made on admission). If the data are normally distributed, one-way analysis of variance (ANOVA) or two-way ANOVA assessing the time interaction between groups and days of observation will be used. When not normally distributed the Kruskal-Wallis test or Friedman test assessing the time interaction between groups and days of observation will be used.

Univariate analysis will be performed to identify potential factors associated with outcomes including, but not limited to, ventilator settings (in particular $\mathrm{V}_{\mathrm{T}}$ and PEEP). A multivariate logistic regression model will be used to determine which of those factors are independent. A stepwise approach will be used to enter new terms into the model, with a limit of $\mathrm{p}<0.2$ to enter the terms. Time to event variables is analysed using Cox regression and visualised by Kaplan-Meier.

Time-course variables (eg, repeated measures of ventilator parameters, vital signs, oxygenation parameters and others) are also analysed by linear mixed model. The linear mixed-models procedure expands the generalised linear model so that the data are permitted to exhibit correlated and non-constant variability.

Prespecified subgroups in the analyses studying potential associations between ventilator settings and outcome will be: (1) patients at low risk of ARDS versus patients at risk of ARDS; (2) patients without ARDS versus patients with ARDS; (3) reason for ICU admission and (4) reason for start of invasive ventilation. Statistical analyses will be conducted using $\mathrm{R}$ (www.r-project.org). A p value of less than 0.05 will be considered statistically significant.

\section{Study organisation}

The Steering Committee is composed of a selection of PROVE Network investigators plus the national coordinators from each participating country. These investigators were involved in the design of PRoVENT-iMIC. National coordinators are responsible for identifying and recruiting local participating centres. They assist and train the local investigators and oversee the conduct of the study, including administrative management, record keeping and data management. Local investigators in individual participating centres will provide scientific and structural leadership, ensuring local ethical and regulatory approvals are obtained before start of patient inclusion. National coordinators and local investigators are expected to guarantee the quality and security of the data collected.

Prior to start of the study, study teams in each centre will undergo a web-based training session on how to capture data in the electronic CRF. All study team members will be provided with a manual of operations with instructions on how to accurately fill the forms and the screening log. Incomplete or incorrectly entered electronic CRFs will be signalled to the local investigators by the national and international coordinator, for further review of the missing or flagged data.

\section{Patient and public involvement}

Patients and public were not directly involved in any phase of this study.

\section{ETHICS AND DISSEMINATION}

Data management, monitoring and reporting of the study will be performed in accordance with the International Conference on Harmonisation-Good Clinical Practice guidelines.

All participating centres will also submit the study protocol to the national or local institutional review board for ethical judgement, as applicable by the current regulations in the country. Due to the strict observational design and anonymous collection of data, informed consent may not be required in most countries. However, where informed consent is required, this must be approved by the local ethical committee before the start of inclusion.

The study will be reported following the Strengthening the reporting of Observational Studies in Epidemiology statement guidelines and checklists. ${ }^{29}$ The results of this study will be published in a peer-reviewed medical journal. After publication of the primary results, on request the pooled dataset will be available for all members of the PRoVENT-iMIC collaboration for secondary analysis, after judgement and approval of scientific quality and validity of the proposed analysis by the Steering Committee.

\section{DISCUSSION}

PRoVENT-iMIC is designed to characterise the epidemiology, ventilator management, occurrence of pulmonary complications and outcomes in invasively ventilated patients in an estimated 60 ICUs in 11 Asian countries. The results of PRoVENT-iMIC will help to understand current ventilation practice in South and Southeast Asia, particularly with respect to variability in ventilator settings among patients without, at risk for or with established ARDS. Results of this study will be used to plan future trials of ventilation in ICU patients in these settings.

PRoVENT-iMIC has several strengths. First, its prospective design will allow a higher accuracy of data capturing with regard to exposures, confounders and endpoints compared with studies that used a retrospective design. ${ }^{30}$ While a prospective design may cause sources of bias and fail to establish causal effects, it minimises the chance of residual confounding by unmeasured variables, a common limitation with a retrospective design, as has frequently been used in mechanical ventilation epidemiological studies. ${ }^{31-33}$ We will have a sample size 
large enough to obtain precise estimates of pulmonary complications and ICU mortality and to examine potential associations between ventilation practice and patient outcomes. Second, the study sample is not restricted to certain patient diagnostic categories. Third, the attention on ventilation practice will provide robust data on this specific domain while the 7 days follow-up will allow precise recording of pulmonary complications at their origin. And finally, the wide representation of Asian countries will allow inferences on geoeconomic differences in epidemiology, management and outcomes of mechanical ventilation across the entire subcontinent.

The focus on South and Southeast Asia follows our scarce knowledge about clinical practices and ventilation strategies used in critically ill patients in this and other resource-limited settings. ${ }^{21}$ The burden of critical illness in low-income and middle-income countries is higher than generally perceived and it is expected to increase with an ageing population. ${ }^{34}$ Additionally, ICUs are increasingly being set up in the region, especially in busy urban settings. A recent survey highlighted considerable variation in structure, organisation and critical care delivery in Asian ICUs, but did not shed light on ventilation management and patient-centred ventilation-associated outcomes. ${ }^{35}$ This information, however, is crucial for future trials of ventilation in ICU patients in these settings, as we need to know whether critically ill patients across Asia equally benefit from lung-protective ventilation as those in Western countries. Additionally, for proper power calculations, information with regard to potential primary endpoints, like the incidence of development of ARDS, duration of ventilation or death, is highly needed. PRoVENT-iMIC will be the first observational study that can provide this information for settings in South Asia. Results restricted to individual settings could also be valuable for local clinicians seeking to improve their local practice, training planning and identify local priorities for quality improvement within their departments.

There is now strong evidence-based support for various ICU process-based interventions such as lung-protective ventilation, ${ }^{36}$ conservative fluid management strategies ${ }^{37}$ and weaning protocols. ${ }^{38}$ While centre-specific or country-specific practices or restrictions of resources are potential challenges that affect implementation of all these interventions, we focus on the management of ventilation and especially on the employment of lung-protective ventilation where feasibility may represent an issue-specific to resource-limited settings. Recent literature has underlined the potential role of the driving pressure (the pressure amplitude during each artificial breath) and its determinants in the development of ventilator-associated lung injury. Results from PRoVENT-iMIC will provide further data to enable us to discriminate the effects of $\mathrm{V}_{\mathrm{T}}$ size, PEEP and driving pressure on outcomes in patients with, at risk of, or without ARDS.

PRoVENT-iMIC will provide important data regarding outcomes following invasive ventilation, including a wide range of clinically important pulmonary complications.
Historical studies from low-resource settings documented mortality rates to exceed $70 \%{ }^{14-16}$ However, more recent data from South America and India have documented mortality rates of $\sim 40 \%$, similar to that in high-income countries. ${ }^{1718}$ This suggests that mortality in ventilated patients has the potential to improve in low-resource settings. ${ }^{12} 1339$ Although many factors may influence mortality, several underappreciated factors related to invasive ventilation may have contributed, including reduced need for invasive ventilation per se, improvements in safety of invasive ventilation and in liberation from invasive ventilation.

Our interest in patients at risk of ARDS follows a global recent shift in ARDS research. It is now clear that ARDS is rarely present at the time of the initial healthcare encounter, and typically develops during the hospital course, usually between days 2 and 5 in patients with predisposing conditions or risk factors. ${ }^{40}$ Hence, increasing efforts are being directed towards early identification of patients at risk with a goal of prevention and early treatment prior to the development of a fully established syndrome. This is probably equally important in resource-limited settings where the predisposing conditions and risk modifiers for ARDS may differ and limited escalation of therapy is often the case. PRoVENT-iMIC will be the first study to evaluate prospectively the role of LIPS in these settings. Although the poor predictive accuracy of the LIPS does not currently support its use in everyday clinical practice, ${ }^{41}$ it has enabled enrolment in clinical trials of ARDS prevention ${ }^{23}$ and may yield an initial idea on the patients at risk of and disease progression in the Asian settings under study.

PRoVENT-iMIC has some noticeable shortcomings. The definition of 'middle-income' country is rather artificial as the level of health expenditure, local resources and other geocultural factors might affect the processes of care in a larger extent than national income classification. Despite the inclusion of ICUs from 11 countries, which improves study generalisability, caution is needed when applying the results to supposedly similar settings, as substantial intranational and transnational variations in ICU resources, staffing and organisation exist. Second, the CRF used in PRoVENT-iMIC was designed so that it would not induce excessive workload for the participating centres. Therefore, we decided not to collect data regarding extrapulmonary complications and hospital discharge outcomes, neither the amounts of sedation used and sedation levels. Similarly, due to the time window restricted to the ICU stay, we will apply the LIPS at ICU admission and not in the first 6 hours after hospital admission, as originally designed. Mortality may be underestimated in some settings where due to local practices there is the possibility to be discharged home in case of terminal conditions or family decision. To address this we made sure the data collection form captures this event whenever it represents the reason of discharge. Third, due to the limitation of laboratory data, we will have a limited dataset that will not comprise daily 
severity scores useful for statistical controlling purposes. Also, the conceivable limitation in blood gas analysis and radiology examinations may limit the documentation of insurgence or worsening of ARDS and other pulmonary complications. Fourth, as in patients on pressure-control modes flow might not reach zero during inspiration, Pmax might overestimate alveolar pressure, hence overestimating driving pressure. An end-inspiratory occlusion could solve this problem, but is almost never performed in many centres. As this study only uses data that is collected as part of standard care, all analysis regarding driving pressure will be done separately for patients on pressure-control modes and volume-control modes. Finally, we cannot exclude that ventilator settings applied by treating physicians might be biased by the participation in the study, a problem that also existed in prior multinational studies. ${ }^{11} 12$ Also participation in international studies like PRoVENT-iMIC always bears the risk of biased to those centres that do not fully or reliably represent ICU care in general in the participating countries.

\section{CONCLUSIONS}

PRoVENT-iMIC is designed to understand the epidemiology, practice of ventilation and outcomes of critically ill patients receiving invasive ventilation in a large set of South Asian countries. Results of this study could help identify practices that may best explain differences in outcomes, and could be used in designing new trials of ventilation in these settings.

\section{Author affiliations}

${ }^{1}$ Department of Intensive Care, Academic Medical Center, Amsterdam, The Netherlands

${ }^{2}$ Mahidol Oxford Tropical Medicine Research Unit (MORU), Faculty of Tropical Medicine, Mahidol University, Bangkok, Thailand

${ }^{3}$ Department of Intensive Care Medicine, Hospital Israelita Albert Einstein, São Paulo, Brazil

${ }^{4}$ Department of Critical Care, BIRDEM General Hospital, Dhaka, Bangladesh

${ }^{5}$ Department of Surgery, Chiang Mai University, Chiang Mai, Thailand

${ }^{6}$ Dev Care Foundation, Chittagong, Bangladesh

${ }^{7}$ National Research Institute of Tuberculosis and Lung Diseases, Shahid Beheshti University of Medical Sciences, Tehran, Iran

${ }^{8}$ Department of Anaesthesiology, Aga Khan University, Karachi, Pakistan

${ }^{9}$ Department of Clinical Tropical Medicine, Faculty of Tropical Medicine, Mahidol University, Bangkok, Thailand

${ }^{10}$ Department of Intensive Care, Sri Jayewardenepura General Hospital, Nugegoda,

Sri Lanka

${ }^{11}$ Department of Medicine, Bharati Vidyapeeth Medical College, Pune, Maharashtra, India

${ }^{12}$ Department of Internal Medicine, Patan Academy of Health Sciences, Kathmandu, Nepal

${ }^{13}$ Department of Critical Care Medicine, St. John's Medical College, Bangalore, India

${ }^{14}$ Department of Intensive Care, Indira Gandhi Memorial Hospital, Malé, Maldives

${ }^{15}$ Oxford University Clinical Research Unit, National Hospital for Tropical Diseases, Hanoi, Vietnam

${ }^{16}$ Critical Care Unit, Ispat General Hospital, Rourkela, Orissa, India

${ }^{17}$ Oxford University Clinical Research Unit, Hospital for Tropical Diseases, Ho Chi

Minh City, Vietnam

${ }^{18}$ Medical Action Myanmar, Naypyidaw, Myanmar

${ }^{19}$ Jeffrey Cheah School of Medicine and Health Sciences, Monash University

Malaysia, Johor, Malaysia
${ }^{20}$ Department of Emergency and Organ Transplantation (DETO), University of Bari, Bari, Italy

${ }^{21}$ Pulmonary Engineering Group, Department of Anesthesiology and Intensive Care Medicine, University Hospital Carl Gustav Carus, Technische Universität Dresden, Dresden, Germany

${ }^{22}$ Department of Surgical Sciences and Integrated Diagnostics, San Martino Policlinico Hospital, IRCCS for Oncology, University of Genoa, Genoa, Italy

${ }^{23}$ Laboratory of Experimental Intensive Care and Anesthesiology (LEICA), Academic Medical Center, Amsterdam, The Netherlands

Collaborators MORU, the Mahidol Oxford Tropical Medicine Research Unit, Bangkok, Thailand (www.tropmedres.ac). PROVE Network, the Protective Ventilation Network (www.provenet.eu).

Contributors LP, AGA, ASN, AMD and MJS were equally responsible for writing of the manuscript and participated in study design. FP and PP participated in study design and assisted in writing of the manuscript. $A A, A B, K C, A F, R a H, R e H, M H, H A l$, $\mathrm{KI}, \mathrm{SI}, \mathrm{GK}, \mathrm{BK}, \mathrm{HM}, \mathrm{BN}, \mathrm{RP}, \mathrm{SS}, \mathrm{LT}, \mathrm{SG}, \mathrm{NNT}$, NMY and MGdA reviewed the manuscript and agreed with submission.

Funding This research received no specific grant from any funding agency in the public, commercial or not-for profit sectors.

Competing interests None declared.

Patient consent Not required.

Ethics approval The Oxford Tropical Research Ethical Committee at the University of Oxford, UK, exempted the study from ethical review on the 9 June 2017.

Provenance and peer review Not commissioned; externally peer reviewed.

Open Access This is an Open Access article distributed in accordance with the Creative Commons Attribution Non Commercial (CC BY-NC 4.0) license, which permits others to distribute, remix, adapt, build upon this work non-commercially, and license their derivative works on different terms, provided the original work is properly cited and the use is non-commercial. See: http://creativecommons.org/ licenses/by-nc/4.0/

(C) Article author(s) (or their employer(s) unless otherwise stated in the text of the article) 2018. All rights reserved. No commercial use is permitted unless otherwise expressly granted.

\section{REFERENCES}

1. Slutsky AS, Ranieri VM. Ventilator-induced lung injury. N Engl J Med 2014;370:980.

2. Serpa N, Schultz M, Slutsky A. Current concepts of protective ventilation during general anaesthesia. Swiss Med Wkly 2015;145:w14211.

3. Putensen C, Theuerkauf N, Zinserling J, et al. Meta-analysis: ventilation strategies and outcomes of the acute respiratory distress syndrome and acute lung injury. Ann Intern Med 2009;151:566-76.

4. Serpa Neto A, Cardoso SO, Manetta JA, et al. Association between use of lung-protective ventilation with lower tidal volumes and clinical outcomes among patients without acute respiratory distress syndrome. JAMA 2012;308:1651.

5. Güldner A, Kiss T, Serpa Neto A, et al. Intraoperative protective mechanical ventilation for prevention of postoperative pulmonary complications. Anesthesiology 2015;123:692-713.

6. Briel M, Meade M, Mercat A, et al. Higher vs lower positive endexpiratory pressure in patients with acute lung injury and acute respiratory distress syndrome: systematic review and meta-analysis. JAMA 2010;303:865-73.

7. Ferguson ND. Low tidal volumes for all? JAMA 2012;308:1689.

8. Serpa Neto A, Nagtzaam L, Schultz MJ. Ventilation with lower tidal volumes for critically ill patients without the acute respiratory distress syndrome: a systematic translational review and meta-analysis. Curr Opin Crit Care 2014;20:25-32.

9. Dellinger RP, Levy MM, Rhodes A, et al. Surviving sepsis campaign: international guidelines for management of severe sepsis and septic shock, 2012. Intensive Care Med 2013;39:165-228.

10. Amato MBP, Meade MO, Slutsky AS, et al. Driving pressure and survival in the acute respiratory distress syndrome. $N$ Engl J Med Overseas Ed 2015;372:747-55.

11. Bellani G, Laffey JG, Pham T, et al. Epidemiology, patterns of care, and mortality for patients with acute respiratory distress syndrome in intensive care units in 50 countries. JAMA 2016;315:788-33. 
12. Neto AS, Barbas CSV, Simonis FD, et al. Epidemiological characteristics, practice of ventilation, and clinical outcome in patients at risk of acute respiratory distress syndrome in intensive care units from 16 countries (PRoVENT): an international, multicentre, prospective study. Lancet Respir Med 2016;4:882-93.

13. LAS VEGAS investigators. Epidemiology, practice of ventilation and outcome for patients at increased risk of postoperative pulmonary complications. Eur J Anaesthesiol 2017;34:492-507.

14. Sinclair JR, Watters DA, Davison M. Outcome of mechanical ventilation in Central Africa. Ann R Coll Surg Engl 1988;70:76-9.

15. Rajapakse VP, Wijesekera S. Outcome of mechanical ventilation in Sri Lanka. Ann R Coll Surg Engl 1989;71:344-6.

16. Sudarsanam TD, Jeyaseelan L, Thomas K, et al. Predictors of mortality in mechanically ventilated patients. Postgrad Med $\mathrm{J}$ 2005;81:780-3.

17. Azevedo LC, Park M, Salluh JI, et al. Clinical outcomes of patients requiring ventilatory support in Brazilian intensive care units: a multicenter, prospective, cohort study. Crit Care 2013;17:R63.

18. Karthikeyan B, Kadhiravan T, Deepanjali S, et al. Case-Mix, care processes, and outcomes in medically-ill patients receiving mechanical ventilation in a low-resource setting from Southern India: a prospective clinical case series. PLoS One 2015;10:e0135336.

19. Serpa Neto A, Schultz MJ, Festic E. Ventilatory support of patients with sepsis or septic shock in resource-limited settings. Intensive Care Med 2016;42:100-3.

20. Haniffa R, Lubell Y, Cooper BS, et al. Impact of a structured ICU training programme in resource-limited settings in Asia. PLoS One 2017; 12:e0173483.

21. Dünser MW, Baelani I, Ganbold L, et al. A review and analysis of intensive care medicine in the least developed countries. Crit Care Med 2006;34:1234-42.

22. The World Bank. World bank country classification. https:// datahelpdesk.worldbank.org/knowledgebase/topics/19280-countryclassification (accessed 17 Dec 2017).

23. Gajic O, Dabbagh O, Park PK, et al. Early identification of patients at risk of acute lung injury: evaluation of lung injury prediction score in a multicenter cohort study. Am J Respir Crit Care Med 2011;183:462-70.

24. Ranieri VM, Rubenfeld GD. The ARDS Definition Task Force. Acute respiratory distress syndrome: the Berlin Definition. JAMA J Am Med Assoc 2012;307:1.

25. Ashizawa K, Hayashi K, Aso N, et al. Lobar atelectasis: diagnostic pitfalls on chest radiography. Br J Radiol 2001;74:89-97.

26. Riviello ED, Kiviri W, Twagirumugabe T, et al. Hospital Incidence and Outcomes of the Acute Respiratory Distress Syndrome Using the Kigali Modification of the Berlin Definition. Am J Respir Crit Care Med 2016;193:52-9.
27. Rice TW, Wheeler AP, Bernard GR, et al. Comparison of the SpO2/ $\mathrm{FIO} 2$ ratio and the $\mathrm{PaO} 2 / \mathrm{FIO} 2$ ratio in patients with acute lung injury or ARDS. Chest 2007;132:410-7.

28. Harris PA, Taylor R, Thielke R, et al. Research electronic data capture (REDCap)--a metadata-driven methodology and workflow process for providing translational research informatics support. J Biomed Inform 2009;42:377-81.

29. von Elm E, Altman DG, Egger M, et al. The Strengthening the Reporting of Observational Studies in Epidemiology (STROBE) statement: guidelines for reporting observational studies. The Lancet 2007;370:1453-7.

30. Euser AM, Zoccali C, Jager KJ, et al. Cohort studies: prospective versus retrospective. Nephron Clin Pract 2009;113:c214-c217.

31. Gajic O, Dara SI, Mendez JL, et al. Ventilator-associated lung injury in patients without acute lung injury at the onset of mechanical ventilation. Crit Care Med 2004;32:1817-24.

32. Rush B, Biagioni BJ, Berger L, et al. Mechanical ventilation outcomes in patients with pulmonary hypertension in the united states: a national retrospective cohort analysis. $J$ Intensive Care Med 2017;32:588-92.

33. Pesaro AE, Katz M, Katz JN, et al. Mechanical Ventilation and Clinical Outcomes in Patients with Acute Myocardial Infarction: A Retrospective Observational Study. PLoS One 2016;11:e0151302.

34. Adhikari NK, Fowler RA, Bhagwanjee S, et al. Critical care and the global burden of critical illness in adults. Lancet 2010;376:1339-46.

35. Arabi YM, Phua J, Koh Y, et al. Structure, organization, and delivery of critical care in Asian ICUs. Crit Care Med 2016;44:e940-e948.

36. Brower RG, Matthay MA, Morris A, et al. Ventilation with lower tidal volumes as compared with traditional tidal volumes for acute lung injury and the acute respiratory distress syndrome. N Engl J Med 2000;342:1301-8.

37. Wiedemann HP, Wheeler AP, Bernard GR, et al. Comparison of two fluid-management strategies in acute lung injury. $N$ Engl J Med 2006;354:2564-75.

38. Girard TD, Kress JP, Fuchs BD, et al. Efficacy and safety of a paired sedation and ventilator weaning protocol for mechanically ventilated patients in intensive care (Awakening and Breathing Controlled trial): a randomised controlled trial. Lancet 2008;371:126-34.

39. Esteban A, Frutos-Vivar F, Muriel A, et al. Evolution of mortality over time in patients receiving mechanical ventilation. Am J Respir Crit Care Med 2013;188:220-30.

40. Gajic O, Dabbagh O, Park PK, et al. Early identification of patients at risk of acute lung injury: evaluation of lung injury prediction score in a multicenter cohort study. Am J Respir Crit Care Med 2010:1-33.

41. Festic E, Kor DJ, Gajic O. Prevention of acute respiratory distress syndrome. Curr Opin Crit Care 2015;21:82-90. 